\title{
SELEÇÃO DE FUNGOS MICORRÍZICOS ARBUSCULARES EFICIENTES PARA PROMOÇÃO DO CRESCIMENTO DA LEGUMINOSA Mimosa artemisiana HERINGER \& PAULA
}

\author{
Andréa Hentz de Mello ; Eliana Maria Ribeiro da Silva²; Orivaldo José Saggin Júnior². \\ ${ }^{1}$ Professora, Doutora da Faculdade de Ciências Agrárias de Marabá, Universidade Federal do Pará - Campus de \\ Marabá. Marabá, Pará, Brasil. andreahentz@ufpa.br \\ ${ }^{2}$ Pesquisadores da Embrapa Agrobiologia. Seropédica, Rio de Janeiro, Brasil.
}

\begin{abstract}
RESUMO: Este trabalho teve como objetivo avaliar a eficiência simbiótica de quatro espécies de fungos micorrízicos arbusculares (FMAs) em promover o crescimento e nutrição da leguminosa Mimosa artemisiana. As sementes foram inoculadas com rizóbio e Glomus clarum (Nicolson \& Schenck), Glomus etunicatum (Becker \& Gerdemann), Gigaspora margarita (Becker \& Hall) e Scutellospora heterogama (Nicol \& Gerd) Walker \& Sanders e o solo foi fertilizado para atingir $5 \mathrm{mg} / \mathrm{kg}$ de P disponível. Glomus clarum foi o fungo que favoreceu o crescimento, os teores de $\mathrm{N}, \mathrm{P}, \mathrm{K}$ na parte aérea e a nodulação da Mimosa artemisiana em relação a testemunha não inoculada, e foi o FMA mais eficiente em beneficiar o crescimento da Mimosa artemisiana, sendo recomendado sua inoculação para a formação de mudas desta leguminosa.
\end{abstract}

PALAVRAS-CHAVE: Gigaspora margarita, Glomus clarum, Glomus etunicatum, micorrizas, Scutellospora heterogama

\section{SELECTION ARBUSCULAR MYCORRHIZAL FUNGI EFFICIENT FOR GROWTH OF LEGUMES Mimosa artemisiana HERINGER \& PAULA}

\begin{abstract}
This study aimed to evaluate the symbiotic efficiency of four AMF species in promove growth and nutrition the Mimosa artemisiana. The seeds were inoculated with rhizobium and arbuscular mycorrhizal fungi Glomus clarum (Nicolson \& Schenck), Glomus etunicatum (Becker \& Gerdemann), Gigaspora margarita (Becker \& Hall) and Scutelospora heterogama (Nicol \& Gerd) Walker \& Sanders in a soil fertilized to achieve $5 \mathrm{mg} / \mathrm{kg}$ and $\mathrm{P}$ disponible. Glomus clarum was the fungi increase growing shoot, N, P, K, contents and nodulation of Mimosa artemisiana the non-innoculated in relation to control and was the most efficient AMF to benefit the growth of Mimosa artemisiana. The inoculation of Glomus clarum is recommended to growth Mimosa artemisiana seedlings.
\end{abstract}

KEY-WORDS: Gigaspora margarita, Glomus clarum, Glomus etunicatum, mycorrhizae, Scutellospora heterogama 


\section{INTRODUÇÃO}

Enquanto a dependência micorrízica (DM) é intrínseca à planta, não sendo afetada pelo ambiente, uma mesma planta pode apresentar diferentes respostas em crescimento e nutrição aos fungos micorrízicos arbusculares (FMAs). Esta variação pode ser devido a diferenças ambientais, sendo o que mais influencia é a disponibilidade de P no solo (KOIDE, 1988).

Quando avaliamos diferentes FMAs em uma mesma planta em condições ambientais padronizadas podemos verificar diferenças no crescimento e nutrição das plantas (SAGGIN JÚNIOR; SIQUEIRA, 1995). Estas diferenças são atribuídas à diferença da funcionalidade da simbiose que é alterada por diferentes combinações fungo-planta. Quando uma espécie de FMA forma uma simbiose funcional com a planta, esta é tida por eficiente e quanto maior a promoção do crescimento e nutrição que a espécie de FMA promova em uma dada planta, mais eficiente esta espécie será (SAGGIN JÚNIOR; SIQUEIRA, 1995).

Como os FMAs não apresentam especificidade em relação ao hospedeiro vegetal (SIQUEIRA, 1986), as variações de resposta da planta às diferentes espécies de FMAs é devido a diferenças de compatibilidade entre os hospedeiros. Certas espécies, tais como Glomus etunicatum e Glomus clarum podem colonizar eficientemente e promover o crescimento de um grande número de espécies vegetais (SAGGIN JÚNIOR; SILVA, 2002), sendo esta característica denominada eficiência simbiótica. Aliado ao fato de que nas florestas tropicais, a diversidade vegetal é muito superior à diversidade de FMAs (FERRER; HERRERA, 1988), a eficiência simbiótica, pode estar relacionada a poucas espécies de FMAs, sendo de grande aplicação tecnológica na formação de mudas, o estabelecimento de combinações fungo-planta eficientes.

Mimosa artemisiana é uma leguminosa arbórea pioneira que tem se destacado no uso para reabilitação de áreas degradadas (SIBINEL, 2003) e arborização de pastagem (FRANCO, 2003 comunicação pessoal). Sendo esta uma espécie que apresenta dependência micorrízica intermediária, (SIBINEL, 2003) é importante à seleção de FMAs eficientes que promoverão $\mathrm{o}$ crescimento e nutrição de suas mudas.

Este trabalho teve como objetivo avaliar a eficiência simbiótica de quatro espécies de FMAs em promover o crescimento $\mathrm{e}$ nutrição de mudas da leguminosa Mimosa artemisiana. 


\section{MATERIAL E MÉTODOS}

$\mathrm{O}$ experimento foi conduzido em casa de vegetação, na Embrapa Agrobiologia, Seropédica - RJ, em copos plásticos de 700 $\mathrm{mL}$, com um tubete acoplado no fundo, totalizando a capacidade de $1000 \mathrm{~mL}$ de substrato por recipiente.

O substrato foi uma amostra de um Latosso Vermelho, textura argilosa, coletado da camada de 0-15 cm, em uma área degradada por empréstimo, utilizada para obras de ampliação da Rodovia RJ 141 que liga Resende à Visconde de Mauá-RJ, pertencente ao Campo de Instrução da Academia Militar das Agulhas Negras. A área localiza-se na encosta da Serrinha do Alambari a $22^{\circ} 22^{\prime} 37^{\prime \prime}$ de Latitude Sul e $44^{\circ} 30^{\prime} 13^{\prime}$ ' de Longitude Oeste, a $550 \mathrm{~m}$ de altitude.

O solo foi passado em peneira de $5 \mathrm{~mm}$ e colocado em cubas contendo $4 \mathrm{~kg}$ de solo. Foi umedecido e autoclavado, permanecendo no autoclave à temperatura de $120^{\circ} \mathrm{C}$ e pressão de $1 \mathrm{~atm}$ por duas horas, repetindo o processo por mais duas vezes, com intervalo de 2 dias, quando apresentou as seguintes características químicas determinadas conforme metodologias descritas pela Empresa Brasileira de Pesquisa Agropecuária (1979): $\mathrm{pH}=4,9 ; \mathrm{Al}=0,5$ $\mathrm{cmol}_{\mathrm{c}} / \mathrm{dm}^{3} ; \mathrm{Ca}+\mathrm{Mg}=0,5 \mathrm{cmol}_{\mathrm{c}} / \mathrm{dm}^{3} ; \mathrm{P}=$
$1 \mathrm{mg} / \mathrm{dm}^{3}$. Antes do plantio, o solo foi fertilizado com P na base de $50 \mathrm{mg} / \mathrm{kg}$, aplicando-se por vaso $25 \mathrm{ml}$ de solução de $\mathrm{KH}_{2} \mathrm{PO}_{4}$ e homogeneizando ao volume total.

Sementes de Mimosa artemisiana Heringer \& Paula, foram escarificadas com $\mathrm{H}_{2} \mathrm{SO}_{4}$ por 3 minutos, para a quebra da dormência, e após lavagem foram inoculadas com rizóbio das estirpes BR 3462 e BR 3609 crescidas em meio semi-sólido descrito por Vicent (1970) e usando turfa esterilizada como veículo antes de serem semeadas diretamente nos recipientes contendo o solo.

Os FMAs utilizados foram Glomus etunicatum (BECKER \& GERDEMANN), Glomus clarum (NICOLSON \& SCHENCK), Gigaspora margarita (BECKER \& HALL) e Scutellospora heterogama (NICOL \& GERD) WALKER $\&$ SANDERS, além de uma testemunha não inoculada, que recebeu um filtrado dos inóculos dos FMAs isento de propágulos. $\mathrm{O}$ delineamento experimental foi em blocos ao acaso, com cinco tratamentos de FMAs e seis repetições.

No orifício de plantio, foram colocadas três sementes inoculadas com rizóbio e foi pipetado sobre as sementes cerca de 90 esporos de cada espécie de fungo micorrízico arbuscular de acordo com o tratamento. Após o plantio as sementes e 
inoculantes foram cobertas com fina camada de solo e irrigadas com $25 \mathrm{~mL}$ de solução nutritiva, aplicando-se por vaso, $1000 \mathrm{mg}$ de $\mathrm{Ca}$ na forma de $\mathrm{CaSO}_{4} ; 35 \mathrm{mg}$ de $\mathrm{N}$ na forma de $\mathrm{NH}_{4} \mathrm{NO}_{3} ; 200 \mathrm{mg}$ de $\mathrm{Mg}$ na forma de $\mathrm{MgSO}_{4} \cdot 7 \mathrm{H}_{2} \mathrm{O} ; 10 \mathrm{mg}$ de $\mathrm{Zn}$ na forma de $\mathrm{ZnSO}_{4} .7 \mathrm{H}_{2} \mathrm{O} ; 10 \mathrm{mg}$ de $\mathrm{B}$ na forma de $\mathrm{H}_{3} \mathrm{BO}_{3} ; 5 \mathrm{mg}$ de $\mathrm{Cu}$ na forma de $\mathrm{CuSO}_{4} .5 \mathrm{H}_{2} \mathrm{O} ; 0,5 \mathrm{mg}$ de $\mathrm{Mo}$ na forma $\mathrm{Na}_{2} \mathrm{MoO}_{4} \cdot 2 \mathrm{H}_{2} \mathrm{O}$.

Esta adubação foi repetida quinzenalmente durante a condução do experimento. Aos 30 dias após a germinação foi realizado um desbaste, deixando uma planta por vaso.

As irrigações foram diárias, de modo a manter a umidade próxima a $70 \%$ do volume total de poros.

Durante o cultivo aos 50, 80 e 110 dias, a Mimosa artemisiana foi avaliada quanto ao crescimento em altura e diâmetro do caule. Aos 140 dias após a germinação, foi colhida e avaliada quanto à altura, diâmetro do caule, número de folhas, número de ramos, massa da parte aérea fresca e seca, densidade de esporos no solo, número de nódulos, teores de nutrientes na parte aérea e colonização micorrízica.

Para o clareamento e coloração das raízes foi utilizada a metodologia de Koske e Gemma (1989) e Grace e Stribley (1991). A colonização micorrízica das raízes foi avaliada pelo método da interseção em placa quadriculada (GIOVANETTI; MOSSE, 1980).

Para a extração dos esporos, foi realizado o peneiramento úmido de $50 \mathrm{~mL}$ de solo (GERDEMANN; NICOLSON, 1963), com posterior centrifugação em água e sacarose a 40\% (JENKINS, 1964). O número de esporos em $50 \mathrm{~mL}$ de solo foi determinado pela contagem dos esporos em microscópio estereoscópico.

O material da parte aérea foi seco a $65^{\circ}$ C em estufa com circulação de ar e pesado até atingir massa constante. Então, foi moído e digerido com extrator nitro-perclórico (2:1) para extração de $\mathrm{P}, \mathrm{K}, \mathrm{Ca}$ e $\mathrm{Mg}$ e extrator sulfúrico para extração de N (SILVA, 1999; LOUREIRO; BODDEY, 1986).

Os teores de $\mathrm{P}$ foram determinados por colorimetria, $\mathrm{K}$ por fotometria de chama, $\mathrm{Ca}$ e $\mathrm{Mg}$ por espectofotometria de absorção atômica (SILVA, 1999) e de N pelo método de Kjeldahl modificado (LOUREIRO; BODDEY, 1986).

Os dados foram testados quanto a sua normalidade e submetidos à análise de variância, teste de médias (Tukey 5\%), utilizando-se dos procedimentos disponíveis no programa Sisvar (FERREIRA, 1998). 
RESULTADOS E DISCUSSÃO

Aos 50, 80, 110 e 140 dias após a germinação da Mimosa artemisiana, os resultados mostram efeitos significativos na altura e diâmetro do colo das mudas (Figura

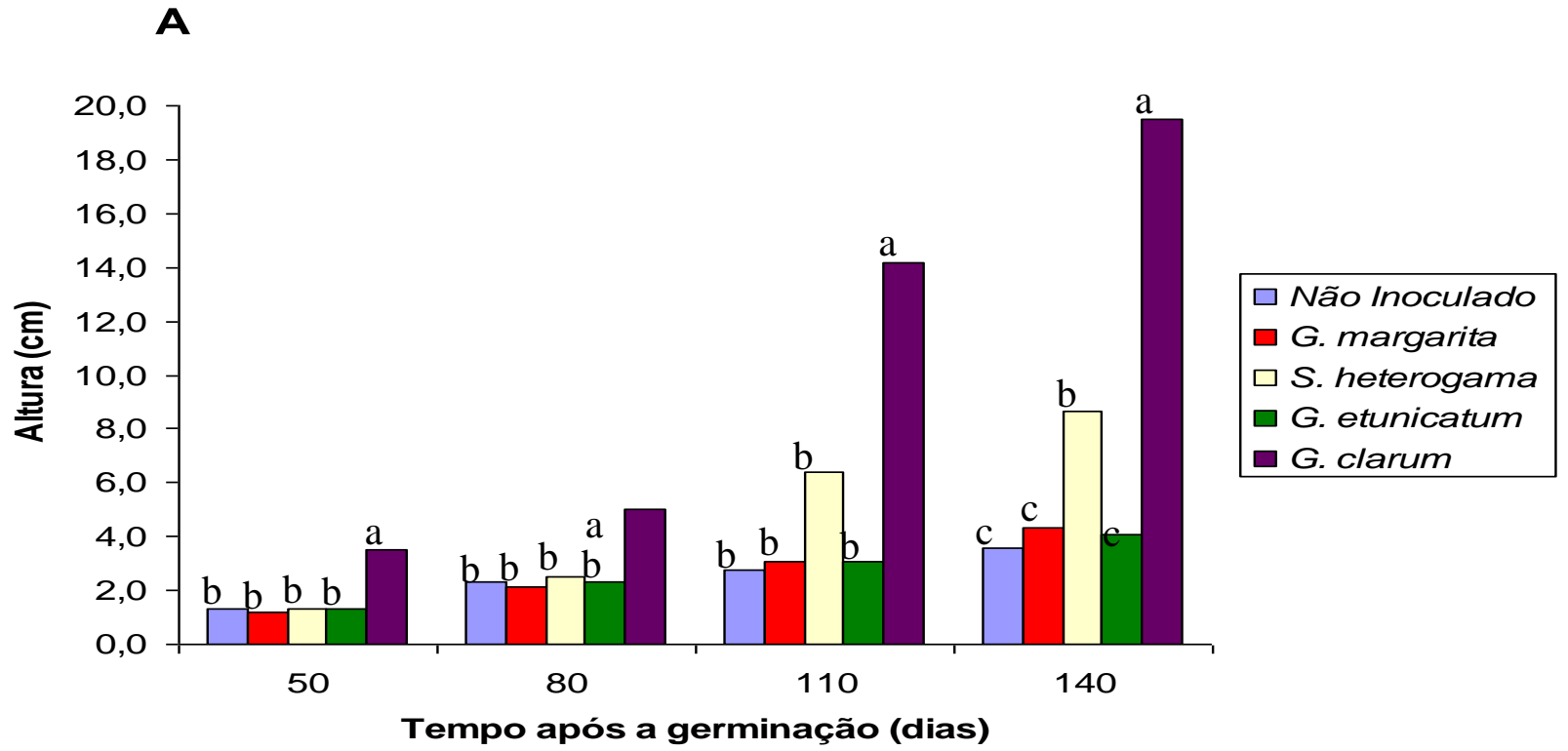

B

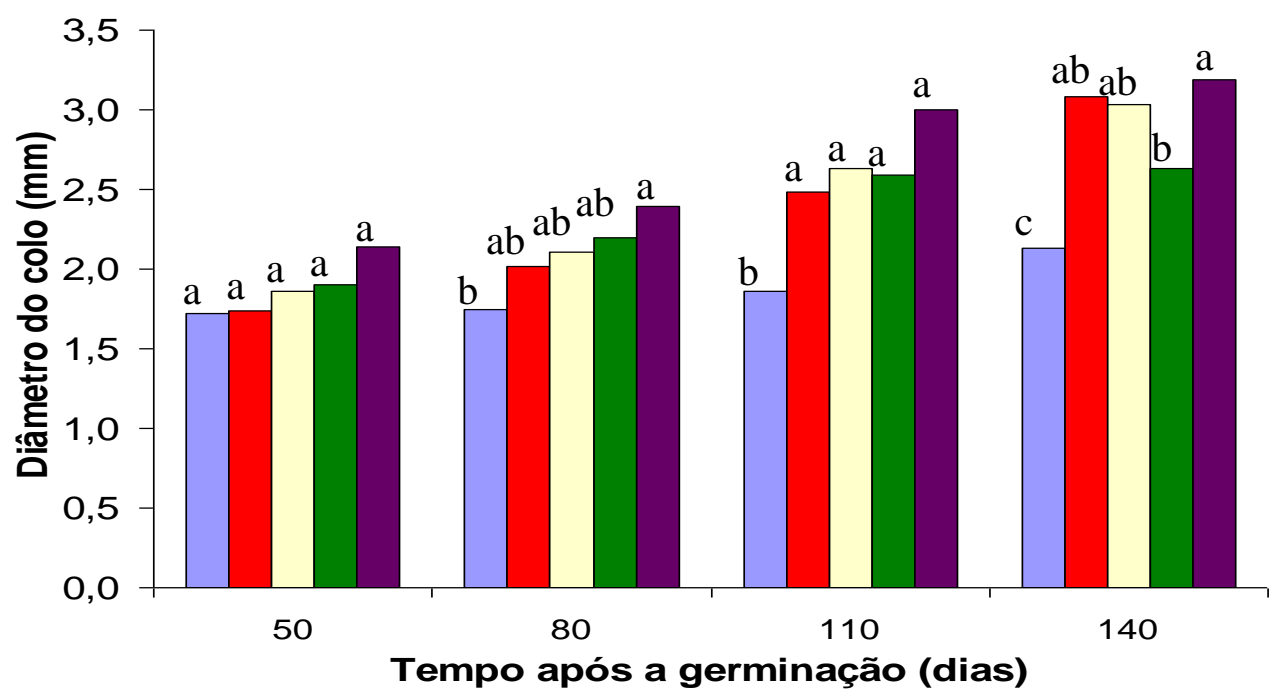

Figura 1. Altura (A) e diâmetro do colo (B) de mudas de Mimosa artemisiana inoculadas com diferentes espécies de FMAs aos 50, 80, 110 e 140 dias após a germinação. Letras iguais dentro de cada época não diferem entre si por Tukey 5\%. 
Glomus clarum mostrou-se mais eficiente que os demais FMAs em promover o crescimento das mudas da Mimosa artemisiana desde a primeira avaliação de altura (Figura 1 A). Ao final do ensaio (aos 140 dias), a altura das mudas de Mimosa artemisiana inoculadas com o fungo Glomus clarum, era mais que o dobro do tratamento de inoculação com Scutelospora heterogama, o que também apresentava crescimento superior ao dos demais FMAs e da testemunha não inoculada.

Em relação ao diâmetro do colo, Glomus clarum mostrou benefício em relação a testemunha não inoculada a partir dos 80 dias após a germinação, entretanto, não diferia dos demais tratamentos de inoculação, exceto aos 140 dias quando apresentou-se mais eficiente em promover o diâmetro do colo que Glomus etunicatum. Todos os tratamentos de inoculação promoveram maior diâmetro do colo que a testemunha não inoculada a partir de 110 dias após a germinação.

A massa da parte aérea seca e fresca, e o número de folhas das mudas de Mimosa artemisiana também foram beneficiadas com a inoculação de Glomus clarum em relação aos demais tratamentos (Tabela 1). Glomus clarum mostrou-se mais eficiente que os demais fungos em promover a produção de massa e o número de folhas.

Tabela 1. Massa da parte aérea seca, massa da parte aérea fresca, número de folhas das mudas da Mimosa artemisiana inoculada com diferentes espécies de fungos micorrízicos arbusculares aos 140 dias após a germinação. (Média de 6 repetições).

\begin{tabular}{|c|c|c|c|}
\hline \multirow[t]{2}{*}{ Tratamento } & Massa da parte aérea seca & Massa da parte aérea fresca & \multirow{2}{*}{$\begin{array}{l}\text { Número de folhas } \\
\text { n } \% \text { planta }^{-1}\end{array}$} \\
\hline & & & \\
\hline Não inoculado & $0,018 \mathrm{c}$ & $0,059 \mathrm{c}$ & $9,8 \mathrm{c}$ \\
\hline Glomus clarum & $0,967 \mathrm{a}$ & $1,839 \mathrm{a}$ & 25,7 a \\
\hline Glomus etunicatum & $0,287 \mathrm{~b}$ & $0,660 \mathrm{~b}$ & $17,2 \mathrm{~b}$ \\
\hline Gigaspora margarita & $0,022 \mathrm{c}$ & $0,056 \mathrm{c}$ & $5,7 \mathrm{c}$ \\
\hline Scutelospora heterogama & $0,340 \mathrm{~b}$ & $0,653 \mathrm{~b}$ & $11,2 \mathrm{bc}$ \\
\hline $\mathrm{CV}(\%)$ & 44,87 & 39,56 & 28,49 \\
\hline
\end{tabular}

Letras iguais nas colunas indicam ausência de diferença entre as médias por Tukey 5\%.

Glomus etunicatum e Scutelospora heterogama também apresentaram benefícios na promoção destas variáveis em relação à testemunha não inoculada. Apenas Gigaspora margarita mostrou-se ineficiente em promover o crescimento de Mimosa artemisiana não diferindo da testemunha não inoculada.

$\mathrm{Na}$ Tabela 2 encontram-se as avaliações microbiológicas de colonização micorrízica, 
número de esporos e número de nódulos por planta.

A esporulação do Glomus clarum e Scutelospora heterogama foram maiores em relação aos demais tratamentos de inoculação.

A esporulação de Glomus clarum foi equivalente a seis vezes a de Glomus etunicatum, e duas vezes a de Gigaspora margarita. Entretanto, não ocorreram diferenças significativas na colonização micorrízica das raízes entre os tratamentos de inoculação.
O número de nódulos também foi promovido pelo FMA mais eficiente (Glomus clarum) em relação aos demais fungos, sendo mais de cinco vezes o número de nódulos da testemunha não inoculada. Scutelospora heterogama também beneficiou o número de nódulos em relação à testemunha, mas os demais fungos não promoveram benefícios da inoculação na nodulação.

Tabela 2. Número de esporos, colonização micorrízicas e número de nódulos das raízes das mudas da Mimosa artemisiana inoculada com diferentes espécies de fungos micorrízicos arbusculares aos 140 dias após a germinação (Média de 6 repetições).

\begin{tabular}{|c|c|c|c|}
\hline Tratamento & $\begin{array}{l}\text { Número de esporos } \\
-n^{\circ} / 50 \mathrm{ml}-\end{array}$ & Colonização micorrízica & $\begin{array}{l}\text { Número de nódulos } \\
-\mathrm{n}^{\circ} / \text { planta }^{-1}\end{array}$ \\
\hline Não inoculado & $0 \mathrm{~b}$ & $0 \mathrm{~b}$ & $2,7 \mathrm{c}$ \\
\hline Glomus.clarum & $211,0 \mathrm{a}$ & $46,3 \mathrm{a}$ & $15,2 \mathrm{a}$ \\
\hline Glomus etunicatum & $35,8 \mathrm{~b}$ & $51,5 \mathrm{a}$ & $2,8 \mathrm{bc}$ \\
\hline Gigaspora margaritta & $87,8 \mathrm{~b}$ & $31,1 \mathrm{a}$ & $2,7 \mathrm{c}$ \\
\hline Scutelospora heterogama & $194,0 \mathrm{a}$ & $39,9 \mathrm{a}$ & $8,0 \mathrm{~b}$ \\
\hline $\mathrm{CV}(\%)$ & 53,91 & 38,47 & 47,99 \\
\hline
\end{tabular}

Letras iguais nas colunas indicam ausência de diferença entre as médias por Tukey 5\%

Os teores de nutrientes na parte aérea das mudas da Mimosa artemisiana são apresentados na Tabela 3. Verifica-se que Glomus clarum, foi o FMA mais eficiente em promover o crescimento de Mimosa artemisiana, promovendo teores de N, P e K maiores que a testemunha não inoculada, sendo que, no caso do $\mathrm{P}$ e $\mathrm{K}$, os teores foram maiores que nos demais tratamentos de inoculação. 
Tabela 3. Teores de $\mathrm{N}, \mathrm{P}, \mathrm{K}, \mathrm{Ca}$ e $\mathrm{Mg}$ das mudas da Mimosa artemisiana inoculada com diferentes espécies de fungos micorrízicos arbusculares aos 140 dias após a germinação (Média de 6 repetições).

\begin{tabular}{|c|c|c|c|c|c|}
\hline Tratamento & $\mathrm{N}$ & $\mathrm{P}$ & $\mathrm{K}$ & $\mathrm{Ca}$ & $\mathrm{Mg}$ \\
\hline & م & & & & \\
\hline Não inoculado & $2,22 \mathrm{c}$ & $1,18 \mathrm{~b}$ & $3,44 \mathrm{~b}$ & $17,88 \mathrm{ab}$ & $3,44 \mathrm{a}$ \\
\hline G. clarum & $2,49 \mathrm{ab}$ & $2,18 \mathrm{a}$ & $9,00 \mathrm{a}$ & $14,45 \mathrm{~b}$ & $3,60 \mathrm{a}$ \\
\hline G. etunicatum & $2,53 \mathrm{a}$ & $1,21 \mathrm{~b}$ & $3,23 \mathrm{bc}$ & $12,58 \mathrm{~b}$ & $3,18 \mathrm{a}$ \\
\hline G. margarita & $2,52 \mathrm{a}$ & $1,38 \mathrm{~b}$ & $2,71 \mathrm{bc}$ & $18,73 \mathrm{ab}$ & $4,78 \mathrm{a}$ \\
\hline S. heterogama & $2,26 \mathrm{bc}$ & $1,30 \mathrm{~b}$ & $1,87 \mathrm{c}$ & $23,29 \mathrm{a}$ & $3,06 \mathrm{a}$ \\
\hline $\mathrm{CV}(\%)$ & 5,92 & 13,91 & 20,31 & 27,83 & 37,10 \\
\hline
\end{tabular}

Letras iguais nas colunas indicam ausência de diferença entre as médias por Tukey 5\%

Em relação aos teores de $\mathrm{N}$, apenas o tratamento de inoculação com Scutelospora heterogama não diferiu da testemunha não inoculada, não refletindo o efeito deste fungo sobre a nodulação das plantas (Tabela 2).Isto sugere que a aplicação de solução nutritiva contendo $\mathrm{N}$ e os diferentes crescimento da planta, afetaram bastante os teores deste nutriente, que em geral foi considerado alto, acima dos níveis comuns para leguminosas (MALAVOLTA, 1989).

$\mathrm{O}$ incremento de $\mathrm{P}$ e $\mathrm{K}$ nas plantas com Glomus clarum foi em média, 1,7 e 3,5 vezes, respectivamente, superior aos demais tratamentos de inoculação os quais não diferiram da testemunha não inoculada. Estes nutrientes foram aplicados em pequenas doses apenas no plantio, e não sendo adicionado posteriormente em solução nutritiva, promovendo as maiores respostas nutricionais de Glomus clarum. Isto indica a eficiência superior da associação Glomus clarum - Mimosa artemisiana de explorar o solo obtendo nutrientes.

$\mathrm{O}$ teor de $\mathrm{Ca}$ foi maior nas plantas inoculadas com Scutelospora heterogama em relação às plantas inoculadas com Glomus clarum e Glomus etunicatum, embora os teores tenham sido elevados em todos os tratamentos. Os teores de $\mathrm{Mg}$ não diferiram entre os tratamentos. Para estes nutrientes, bastante móveis na solução do solo e fornecidos pela solução nutritiva, esperava-se pouco efeito dos tratamentos de inoculação.

Glomus clarum foi o FMA mais eficiente para Mimosa artemisiana, pois promoveu maiores benefícios no crescimento, nas variáveis analisadas, tais como nodulação e teores de $\mathrm{N}, \mathrm{P}, \mathrm{K}$ na parte aérea. A boa compatibilidade funcional dos simbiontes é confirmada pela alta esporulação do fungo na rizosfera. Scutelospora heterogama seguido de 
Glomus etunicatum também apresentaram certa eficiência em promover o crescimento de Mimosa artemisiana, porém, em algumas variáveis não diferiram do controle, sendo, portanto, menos eficientes. Gigaspora margarita, na maior parte das variáveis, não respondeu diferentemente da testemunha não inoculada sugerindo baixa compatibilidade funcional com Mimosa artemisiana e consequentemente baixa eficiência simbiótica da associação formada.

As diferenças de eficiência verificadas parecem ser devido à habilidade maior da combinação Mimosa artemisiana - Glomus clarum em absorver nutrientes como $\mathrm{P}$ e $\mathrm{K}$ e promover a nodulação, já que não houve diferenças acentuadas na colonização micorrízica. A melhor compatibilidade entre os hospedeiros podem levar a um maior enlongamento de raízes no solo (EDATHIL et al., 1996), podendo haver maior produção de hormônios vegetais (ALLEN et al., 1980) ou alteração na fisiologia na condutância das folhas, alterando a eficiência fotossintética e de transpiração (BETHLENFALVAY et al., 1989). O desenvolvimento da colonização micorrízica e da esporulação na rizosfera das mudas inoculadas com FMAs é função de vários fatores entre eles, espécie florestal, nutrição, substrato, tipo de fungo micorrízico e $\mathrm{pH}$ do solo, mas sendo de grande importância a compatibilidade funcional entre os simbiontes, na formação da simbiose, que leva à eficiência simbiótica do FMA.

Caldeira et al. (1999) observaram que as maiores percentagens de colonização micorrízica em Copaifera martii foram com Glomus clarum e Gigaspora margarita. Mudas de Dimorphandra macrostachya inoculadas com Gigaspora margarita e fungos nativos foram as que tiveram as maiores taxas de colonização micorrízica.

Isto deve-se ao fato dos FMAs terem sido na natureza mais selecionados para a sobrevivência do que para eficiência (MOREIRA; SIQUEIRA, 2002).

As diferenças de eficiência dos FMAs em beneficiar as plantas são verificadas facilmente quando várias espécies de FMAs são inoculadas em uma mesma planta, sendo que estas diferenças podem existir até dentro de isolados de uma mesma espécie de FMA (SAGGIN JÚNIOR; SIQUEIRA, 1995; BETHLENFALVAY et al., 1989).

No presente estudo, Glomus clarum por sua alta eficiência em beneficiar mudas de Mimosa artemisiana tem alto potencial para ser inoculado na formação de mudas desta espécie e de promover o crescimento das plantas em campo, embora em campo outros fatores influenciarão também a eficiência de 
Glomus clarum, tais como competição com outros FMAs e adaptação ao solo e clima do local (ABBOT; ROBSON, 1992).

\section{CONCLUSÃO}

Glomus clarum apresenta alta eficiência simbiótica em promover crescimento, nutrição e nodulação de Mimosa artemisiana com P disponível no solo de $5 \mathrm{mg} / \mathrm{kg}^{-1}$.

Scutelospora heterogama e Glomus etunicatum também apresentam, em menor grau, eficiência em promover o crescimento de Mimosa artemisiana nesta condição de solo.

Gigaspora margarita forma simbiose não compatível funcionalmente com Mimosa artemisiana.

Glomus clarum é recomendado para inoculação de mudas de Mimosa artemisiana

\section{REFERÊNCIAS}

ABBOTT, L.K.; ROBSON, A.D.; GAZEY, C. Selection of inoculant vesiculararbuscular mycorrhizal fungi. In: NORRIS, J.R.; READ, D.J.; VARMA, A.K. (eds) Methods in microbiology. Volume 24 techniques for the study of mycorrhiza. San Diego: Academic press, 1:1-22, 1992, $433 p$.

ALLEN, M. F.; MOORE Jr., T. S.; CHRISTENSEN, M. Phytohormone changes in Baoutelona gracilis infected by vesiculararbuscvular mucorrhizae I. Cytokinin increase in host plant. Can. J. Bot., v. 58, p. 371-374, 1980.

BETHLENFALVAY, G.J.; FRANSON, R.L.; BROWN, M.S.; MIHARA, K.L. The Glycine-Glomus-Bradyrhizobiun symbiosis. IX Nutricional, morphological and physiological responses of nodulated soybean to geografic isolates of the mycorrhizal fungus Glomus mosseae. Physiol. Plant. Copenhagen, v. 76, n. 2, p. 226-32, Jun.1989.

CALDEIRA, W,V,M; SILVA, R.E; FRANCO A.A. Comportamento de mudas de leguminosas arbóreas inoculadas com fungos micorrízicos arbusculares. Ciência Florestal, v.9, n.1, p.135-142, 1999.

EDATHIL, T.T.; MANIAN, S.; UDAIY, K. Interation of multiple VAM fungal species on root colonization, plant grown and nutrient status of tomato seedlings (Lycopersicon esculentum Mill). Agriculture, Ecossystems and Environment, v. 59, p. 61-68, 1996.

FERRER, R.L.; HERRERA, R.A. Micotrofia em Sierra del Rosario. In: HERRERA， R.A; MENENDEZ, L.; RODRIGUEZ, M.E.; GARCIA, E.E. 
Ecoloia de los bosques siempreverdes de la Sierra del Rosario, Cuba: Proyecto MAB n.1, 1974-1987. La Habana: Instituto de Ecologia y Sistematica/Academia de Ciencias en Cuba, 1988. p. 473-484.

FERREIRA, D. F. Sistema de análise estatística para dados balanceados. Lavras: UFLA/DEX/SISVAR, 1998. 145 p,

GERDEMANN, J. W.; NICOLSON, T. H. Spores of mycorrhizal Endogone species extracted from soil by wet sieving and decanting. Transactions of the British Mycological Society, Cambridge, v. 46, p.235-244, 1963.

GIOVANETTI, M.; MOSSE, B. Na evaluation of techniques for measuring VA mycorrhizal infection in roots. The New Phytologist, Oxford, v.84, p.489-500, 1980.

GRACE, C.; STRIBLEY, D. P. A safer procedure for roution staining of vesiculararbuscular mycorrhizal fungi. Mycological Research, Cambridge, v.95, n.10, p.11601162, 1991.

JENKINS, W. R. A rapid centrifugalfloration technique for separatin nematodes from soil. Plant Dis. Rep., v. 48, p. 692, 1964.
KOIDE, R. T. Nutrient supply, nutrient demand and plant response to mycorrhizal infection. New Phytol, v. 117, p. 365-386, 1988.

KOSKE, R. E.; GEMMA, J. N. A modified procedure for stining roots to detect VA mycorrhizas. Mycological Research, Cambridge, v.92, n.4, p.486-488, June 1989.

MALAVOLTA, E. Avaliação do estado nutricional das plantas: princípios e aplicações. Piracicaba: Associação Brasileira para Pesquisa da Potassa e do Fósforo, 1989. $201 \mathrm{p}$.

SAGGIN JÚNIOR, O. J.; SIQUEIRA, J. O. Avaliação da eficiência simbiótica de fungos endomicorrízicos para o cafeeiro. Revista brasileira de Ciência do Solo, Campinas, v.19, n.2, p.221-228, maio/ago. 1995.

SIBINEL, A. H. M. Resposta da leguminosa Mimosa artemisiana à inoculação de diferentes fungos micorrízicos arbusculares na recuperação de áreas degradadas. 57 f. Dissertação (Mestrado em Ciências do Solo) Universidade Federal Rural do Rio de Janeiro. Instituto de Agronomia. 2003.

SILVA, F. C. Manual de análises químicas de solos, plantas e fertilizantes. Brasília: 
Embrapa Solos, Embrapa Informática Agropecuária, 1999.

SIQUEIRA J. O. et al. Mycorrhizal fungi and superphosphate on growth of tropical woody species. Scientia Forestalis, n.50, p.21-36, dez. 1996.
VICENT, J. M. A manual for the practical study of root-nodule bacteria. Oxford Blackwell scientific publications, Oxford, $164 p, 1970$. 